\title{
PAPILEDEMA ASOCIADO A SÍNDROME DE POEMS
}

\section{PAPILLEDEMA AS AN INDICATOR OF POEMS SYNDROME}

\author{
BARNÉS C ${ }^{1}$, SÁNCHEZ-DALMAU BF ${ }^{2}$, VELA D $^{3}$, ALARCÓN S ${ }^{4}$
}

\begin{abstract}
RESUMEN
Caso clínico: Se describe el caso de una paciente de 54 años con papiledema bilateral asociado a síndrome de POEMS. En el estudio se determinó la presencia de hipertensión intracraneal y se instauró tratamiento con acetazolamida.

Discusión: La manifestación oftalmológica del POEMS suele ser el papiledema, cuya etiología puede ser infiltrativa, por hipertensión intracraneal, inflamatoria y por aumento de la permeabilidad vascular. El correcto estudio y tratamiento del edema de papila, según su diferente etiología, permitirá el control de la clínica visual del paciente.
\end{abstract}

Palabras clave: POEMS, papiledema, gammapatía monoclonal, neuropatía periférica, mieloma múltiple, rituximab.

\section{INTRODUCCIÓN}

El síndrome de POEMS es una rara entidad sistémica relacionada con el mieloma múltiple y se define por la presencia de: polineuropatía (sensitivomotora de tipo mixto y predominio desmielinizante), organomegalia, endocrinopatía, componente mono-

\begin{abstract}
Case report: We present the case of a 54-year-old woman with papilledema associated to POEMS syndrome. The presence of intracranial hypertension was detected and treatment started with acetazolamide.

Discussion: The most common ophthalmological pathology in POEMS syndrome is papilledema, the etiology of which could be infiltrative, intracranial hypertension, inflammation or an increase of the vascular permeability. The correct diagnosis and treatment of papilledema, depending on its etiology, should permit an acceptable visual outcome to be achieved (Arch Soc Esp Oftalmol 2008; 83: 709712).
\end{abstract}

Key words: POEMS, papilledema, monoclonal gammopathy, peripheral neuropathy, multiple myeloma, rituximab.

clonal (gammapatía monoclonal $\operatorname{IgA}-\lambda$ o $\operatorname{IgG}$ ) y lesiones cutáneas (hiperpigmentación difusa, acropaquia).

Según los criterios establecidos por Dispienzieri se requieren dos criterios mayores (polineuropatía y componente monoclonal) y al menos un criterio menor (lesiones óseas escleróticas, enfermedad de

Recibido: 23/8/07. Aceptado: 10/11/08.

${ }^{1}$ Licenciado en Medicina. Servicio de Oftalmología del Hospital Mutua de Terrassa. Terrassa. Barcelona. España.

${ }^{2}$ Licenciado en Medicina. Jefe de la Unidad de Neuro-Oftalmología del Institut Clínic d'Oftalmologia (ICOF). Hospital Clínic i Provincial de Barcelona. Barcelona. España.

${ }^{3}$ Licenciadoa en Medicina. Médico del Servicio de Oftalmología de Granollers. España.

${ }^{4}$ Licenciado en Medicina. Médico adjunto del Hospital Mutua de Terrassa. Terrassa. Barcelona. España.

Correspondencia:

Carlos Barnés Ruz

C/. Marqués de Campo Sagrado, $30,4 .^{\circ} 1 .^{\text {a }}$

08015 Barcelona

España

E-mail: 38665cbr@comb.es 
Castleman, organomegalia, edema, endocrinopatía, cambios cutáneos o papiledema) para el diagnóstico de síndrome de POEMS (2).

\section{CASO CLÍNICO}

Paciente de 54 años de edad que presenta parestesias en extremidades inferiores y papiledema bilateral, mayor en ojo izquierdo (OI) que en ojo derecho (OD) y con hemorragias en capa de fibras nerviosas en OI (fig. 1). La paciente refería pérdidas visuales transitorias aunque al diagnóstico se encontraba oftalmológicamente asintomática, con una agudeza visual (AV) de 1.0 en ambos ojos y una campimetría computerizada SITA Standard 24:2 normal. Se había practicado una resonancia magnética nuclear (RMN), que fue normal y un electromiograma (EMG) con polineuropatía mixta. En la analítica se halló una gammapatía monoclonal $\operatorname{Ig} \mathrm{A}-\lambda$ y en la exploración cutánea se observaron áreas de hiperpigmentación y acropaquia (fig. 2). Fue diagnosticada de enfermedad de POEMS y se inició tratamiento con Melfalan $250 \mu \mathrm{g} / \mathrm{kg} /$ día durante 5 días cada 6 semanas y prednisona $30 \mathrm{mg}$ cada 48 horas que se mantuvo durante 1 año.

En 2000 presentó una disminución de la AV. La exploración objetivó una agudeza visual máxima corregida de 0,5 en cada ojo, asociada a un defecto pupilar afererente relativo (DPAR) en el OD. Esto no es frecuente en el papiledema asociado a POEMS pero no existía ninguna otra neuropatía óptica asociada.También se apreció afectación de la visión de colores en el mismo ojo (4/15 en el test de

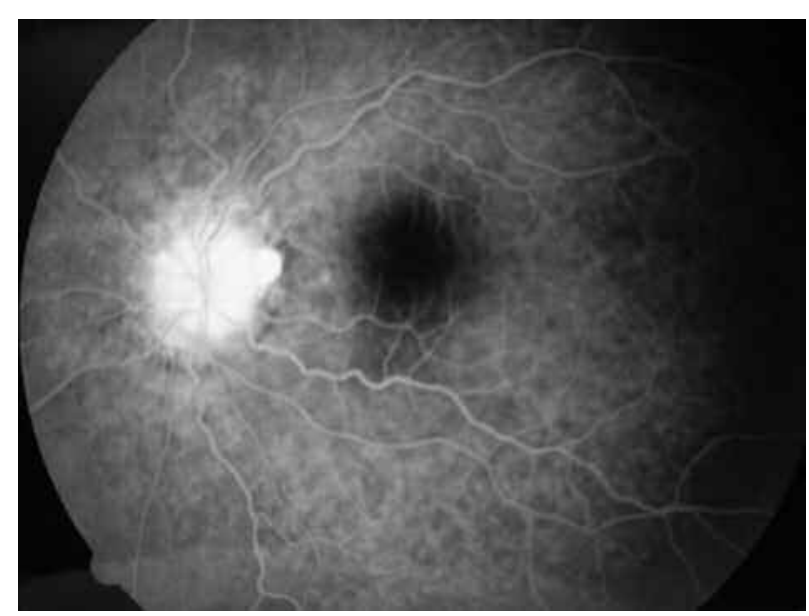

Fig. 1: Papiledema en ojo izquierdo.

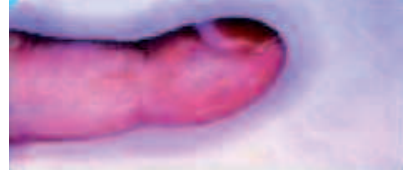

Fig. 2: Acropaquia. Dedo en palillo de tambor.

Ishihara) y una progresión del edema de papila. En el campo visual se evidenció aumento de la mancha ciega bilateral y un escotoma arciforme en OI (fig. 3).

La presión del líquido cefalorraquídeo era $28 \mathrm{cmH}_{2} \mathrm{O}$ (valores normales inferiores a $25 \mathrm{cmH}_{2} \mathrm{O}$ ). Ante la confirmación de hipertensión intracraneal se instauró tratamiento con acetazolamida $250 \mathrm{mg}$ cada 8 horas durante 1 semana, tras la cual se normalizó la presión intrarraquídea.

Al cabo de 1 mes el cuadro sistémico empeoró por lo que el servicio de hematología propuso un trasplante de médula ósea, que la paciente rechazó. Se decidió sustituir el tratamiento con Melfalán por Rituximab, un anticuerpo monoclonal anti-Ag CD20.

La última exploración, practicada tres meses después del tratamiento con Rituximab, mostró una $\mathrm{AV}$ corregida de la unidad en ambos ojos (AO), un DPAR en OD, una afectación parcial de la visión de los colores verde y rojo y, a la evaluación perimétrica mostraba, un aumento de la mancha ciega en AO con un escalón nasal en OI (fig. 4). En el fondo de ojo se apreciaba un edema de papila crónico bilateral. El cuadro visual se hallaba controlado sin necesidad de tratamiento.

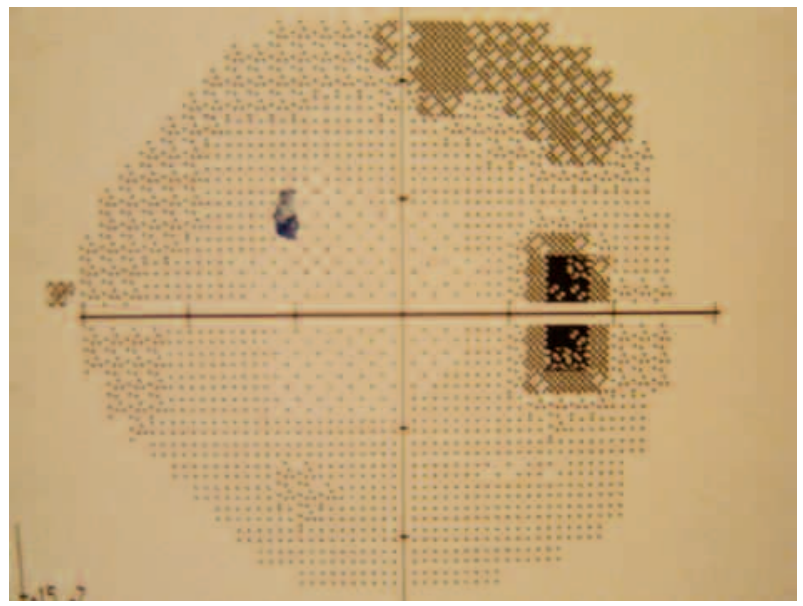

Fig. 3: Aumento de la mancha ciega y defecto arciforme superior en el campo visual del ojo derecho. 


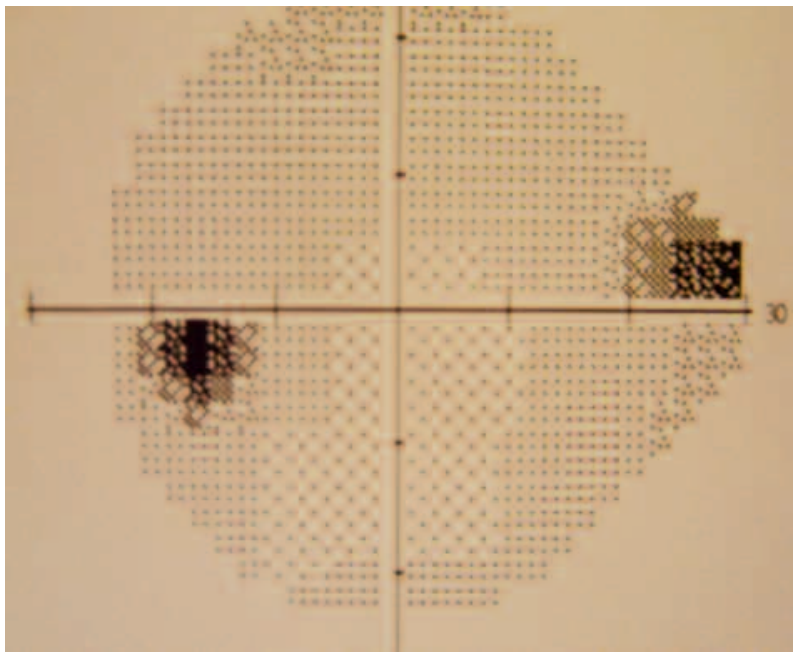

Fig. 4: Escalón nasal en el campo visual del ojo izquierdo.

\section{DISCUSIÓN}

En raras ocasiones el papiledema es la manifestación inicial de enfermedades sistémicas como el síndrome de Guillain-Barré o el síndrome de POEMS [1].

A nivel oftalmológico, pueden presentar papiledema (37-73\%) (2), drusas peripapilares y/o neovascularización coroidea peripapilar. El edema de papila suele ser crónico bilateral con aumento de la mancha ciega, con o sin afectación de la AV (según grado evolutivo), pero sin evolución a atrofia óptica.

Con respecto a la patogenia-fisiopatología, existen diversas teorías: fenómeno de vasculitis en la papila $(2,5)$, infiltración del nervio óptico $(2,5)$, hiperproteinorraquia e hipertensión intracraneal $(2,5) \mathrm{y}$, por último, aumento de la permeabilidad vascular mediado por citoquinas (3).

Hasta la actualidad los tratamientos más habituales eran la radioterapia (sobre lesiones osteoescleróticas localizadas y plasmocitomas), los agentes quelantes (melfalán y ciclofosfamida) y los corticoides, siendo desplazados en este momento por terapias más específicas tal como los anticuerpos monoclona- les como el rituximab (anti CD20) en dosis de 4 infusiones de rituximab $375 \mathrm{mg} / \mathrm{m}^{2}$ en intervalos semanales (4) o los anti VEGF como el bevacizumab.

El tratamiento del edema de papila sólo será necesario en caso de clínica visual y dependerá de la etiología. Ante un paciente con síndrome de POEMS y edema de papila debe realizarse una punción lumbar para conocer su presión intracraneal. Si es superior a $25 \mathrm{~cm}$ de $\mathrm{H}_{2} \mathrm{O}$ consideraríamos que existe hipertensión intracraneal y que ésta sería la causa del papiledema. Entonces realizaremos tratamiento con acetazolamida vía oral hasta la estabilización clínica. Caso de no mejorar, se plantearía la posibilidad terapéutica de realizar una fenestración de la vaina del nervio óptico, aunque en nuestro conocimiento no constan casos que precisaran fenestración en este tipo de pacientes. Si la presión intracraneal fuera inferior a $25 \mathrm{~cm}$ de $\mathrm{H}_{2} \mathrm{O}$ se consideraría que el edema de papila sería secundario a un proceso infiltrativo y se valorarían diversas opciones terapéuticas entre las que se hallan los corticoides sistémicos a altas dosis o la braquiterapia sobre el nervio óptico o el uso más reciente de anticuerpos monoclonales (5).

\section{BIBLIOGRAFÍA}

1. Arruga J, Sánchez Dalmau BF. En: Arruga J, Sánchez B. Neuropatías ópticas: Diagnóstico y tratamiento. 78 Ponencia oficial de la Sociedad Española de Oftalmología. Madrid: 2002; 291-304.

2. Dispenzieri A, Kyle RA, Lacy MQ, Rajkumar $S$, Therneau $T M$, Larson DR, et al. POEMS syndrome: definitions and long-term outcome. Blood 2003; 101: 2496-2506.

3. Martí-Martínez S, Martín-Estefanía C, Turpín-Fenoll L, Pampliega-Pérez A, Reus-Bañuls S, García-Barragan N, et al. Edema de papila bilateral como manifestación inicial de un síndrome de POEMS. Rev Neurol 2006; 43: 531-534.

4. Chong DY, Comer GM, Trobe JD. Optic disc edema, cystoid macular edema, and elevated vascular endothelial growth factor in a patient with POEMS syndrome. J Neuroophthalmol 2007; 27: 180-183.

5. Miralles GD, O'Fallon JR, Talley NJ. Plasma-cell dyscrasia with polyneuropathy: the spectrum of POEMS syndrome. N Eng J Med 1992; 327: 1919-1923. 